\title{
Calculations of longitudinal and transverse velocity structure functions using a vortex model of isotropic turbulence
}

\author{
Guowei $\mathrm{He}^{\mathrm{a})}$ \\ Theoretical Division and Center for Nonlinear Studies, Los Alamos National Laboratory, Los Alamos, \\ New Mexico 87545 and Laboratory for Nonlinear Mechanics of Continuous Media, Institute of Mechanics, \\ Chinese Academy of Sciences, Beijing 100080, China
}

Gary D. Doolen and Shiyi Chen

Theoretical Division and Center for Nonlinear Studies, Los Alamos National Laboratory, Los Alamos, New Mexico 87545

(Received 23 September 1998; accepted 7 September 1999)

\begin{abstract}
The longitudinal structure function (LSF) and the transverse structure function (TSF) in isotropic turbulence are calculated using a vortex model. The vortex model is composed of the Rankine and Burgers vortices which have the exponential distributions in the vortex Reynolds number and vortex radii. This model exhibits a power law in the inertial range and satisfies the minimal condition of isotropy that the second-order exponent of the LSF in the inertial range is equal to that of the TSF. Also observed are differences between longitudinal and transverse structure functions caused by intermittency. These differences are related to their scaling differences which have been previously observed in experiments and numerical simulations. (c) 1999 American Institute of Physics.
\end{abstract}

[S1070-6631(99)02512-X]

\section{INTRODUCTION}

One of the central challenges in fluid turbulence is to understand velocity structure functions. Velocity structure functions are defined as moments of the velocity differences (or velocity increments) between two points in a turbulent field. The longitudinal structure functions (LSFs) are the moments of the differences in the velocity components which are in the same direction as the separation vector between the two points. The transverse structure functions (TSFs) are the moments of the differences in the velocity components which are transverse to the separation vector between the two points. In Kolmogorov's 1941 paper on isotropic turbulence, ${ }^{1}$ the probability distribution functions of the velocity differences between two points were assumed to be independent of coordinate translations and invariant with respect to rotations and reflections of the coordinate systems. Therefore, tensorial moments of velocity differences are homogeneous and isotropic. Using dimensional analysis, Kolmogorov argued that the velocity structure functions are scale invariant in the inertial subrange and he obtained the linear scaling exponents of the LSFs.

Since Kolmogorov's 1941 paper, much work has been done on the calculation of velocity structure functions. Due to experimental constraints, most of the work was focused on the LSFs by invoking Taylor's hypothesis. Experiments and direct numerical simulation (DNS) have revealed that the scaling exponents of the LSFs deviate from Kolmogorov's prediction of linear scaling. The deviations from the linear scaling exponents are a measure of the intermittency of the

a) Author to whom correspondence should be addressed. Telephone: 505667-0174; Fax: 505-665-3003; electronic mail: ghe@t13.lanl.gov
LSFs. (See the review by Sreenivasan and Antonia. ${ }^{2}$ ) Only recently has it become feasible to calculate reliably other components of velocity structure functions, due to the advances in experimental technology and computer capability. Several experimental studies and $\mathrm{DNS}^{3-12}$ have been carried out to determine the TSFs. Most of these results ${ }^{3,6,7,9-12}$ agree that there exist significant differences between scaling exponents of LSFs and those of TSFs for moment orders higher than three. The TSFs exhibit more intense intermittency than the LSFs. However, there also exist some different conclusions, ${ }^{4,5,8}$ which argue that the differences of scaling exponents in experiments and DNS violate the isotropic constraint and attribute to the effects to finite scaling range. ${ }^{13}$

A minimal condition for isotropic turbulence is that the scaling exponents for the second-order LSF and TSF must be the same. It is known that inertial range isotropy can only be obtained for flows with high Reynolds numbers. The existing DNS can only reach moderate Reynolds numbers, where the minimal condition is hardly satisfied. Therefore, theoretical calculations of LSFs and TSFs using various models of isotropic turbulence are of great interest.

The purpose of this paper is to propose a vortex model and use it to calculate velocity structure functions. In Sec. II, a vortex model combining Burgers and Rankine vortices is constructed for isotropic turbulence. Using this model, we derive the related LSFs and TSFs in Sec. III and calculate their scaling exponents in Sec. IV. Finally, we discuss the results and briefly present our conclusions.

\section{A COMBINED VORTEX MODEL FOR ISOTROPIC TURBULENCE}

The basic idea in vortex models of fluid turbulence is that the statistics of turbulence can be calculated using a 
stochastic distribution of various vortices, subject to the required probability density distributions. If vortex solutions of the Navier-Stokes equations specify the essential physics of turbulence and if the vortex distributions resemble the statistics of the observed vortices, the vortex models can reasonably describe the statistics of turbulence. Using vortex models, one can calculate velocity structure functions by ensemble-averaging various vortex solutions. In Synge and Lin's pioneering work, ${ }^{14}$ Hill's spherical vortex was used to calculate the LSFs and TSFs of second order. Since Hill's vortex is inviscid and steady, it is more appropriate for largescale rather than small-scale statistics. A more appropriate vortex solution for small-scale statistics is the Burgers vortex..$^{15}$ It describes the properties of filaments which are the most intermittent structures at small scales in turbulence. ${ }^{16,17}$

The velocity field of the Burgers vortex is composed of a nonrotational strain background field and a field induced by a localized vortex filament. In cylindrical coordinates $(r, \theta, z)$, the background straining velocity field, $\mathbf{v}_{B, b}$, has the following form:

$$
\mathbf{v}_{B, b}=(-a r, 0,2 a z),
$$

where the strain rate, $a$, is a positive constant. The localized vortex filament is assumed to be aligned with the $z$ axis and has the vorticity

$$
\omega=\left(0,0, \omega_{z}(r)\right), \quad \omega_{z}(r)=\frac{\Gamma}{\pi r_{b}^{2}} \exp \left(-\hat{r}^{2}\right),
$$

where $r_{b}=(2 \nu / a)^{1 / 2}$ is the vortex radius, $\Gamma$ is the vortex circulation, $\mathrm{V}$ is the viscosity and $\hat{r}=r / r_{b}$. The velocity associated with $\omega, \mathbf{v}_{B, f}$, has only an azimuthal component

$$
\mathbf{v}_{B, f}=\left(0, \mathbf{v}_{\theta}, 0\right), \quad \theta_{\theta}=\frac{\Gamma}{2 \pi r_{b}} \frac{1-\exp \left(-\hat{r}^{2}\right)}{\hat{r}} .
$$

The total velocity for the Burgers vortex is $\mathbf{v}_{B}=\mathbf{v}_{B, b}+\mathbf{v}_{B, f}$.

Each Burgers vortex can be characterized by two parameters: the vortex radius $r_{b}$ and the vortex strength $\Gamma$. The latter defines the vortex Reynolds number, $R_{\Gamma}=\Gamma / \mathrm{v}$. Assuming that $R_{\Gamma}$ and $r_{b}$ have uniform distributions, Saffman and Pullin calculated the LSFs. ${ }^{18}$ Their results have a satisfactory inertial range for even order LSFs and a limited inertial range for odd order LSFs. An exponential probability density function (PDF) for $R_{\Gamma}$ has been proposed recently by Hatakeyma and Kambe ${ }^{19}$

$$
P\left(R_{\Gamma}\right)=\frac{C^{3}}{2} R_{\Gamma}^{2} \exp \left(-C R_{\Gamma}\right),
$$

where $C=(3 / 4 \pi) R_{\lambda}^{1 / 2}$ and $R_{\lambda}$ is the Taylor-Reynolds number. The LSFs obtained from their calculation show good agreement with experimental and DNS results. Following Hatakeyma and Kambe's assumption, we have further calculated TSFs, but the TSFs obtained do not demonstrate satisfactory inertial range scalings.

A filament can be also modeled by a Rankine vortex. ${ }^{20}$ The Rankine vortex is a uniform rectilinear filament in ideal flows, which is composed of rotational and irrotational parts. The vorticity is assumed to be aligned in parallel straight vortex lines, for example, along the $z$ axis in a cylindrical coordinate system. The induced velocity fields $\mathbf{v}_{R}$ $=\left(0, \mathrm{v}_{R, \theta}, 0\right)$ only have an azimuthal component

$$
\mathrm{v}_{R, \theta}= \begin{cases}\frac{\Gamma}{2 r_{R}^{2}} r & r \leqslant r_{R}, \\ \frac{\Gamma}{2 r}, & r>r_{R},\end{cases}
$$

where $\Gamma$ is the circulation and $r_{R}$ is the core radius. Kambe and Hosokawa were the first to use the Rankine vortex model to calculate the first and third LSFs and their results were impressive. ${ }^{21}$ It has been known that the Rankine vortex is an exact solution of the Navier-Stokes equation in the limit of vanishing viscosity. Therefore, it is an approximation to the concentrated vortices in turbulence at very high Reynolds number. It is the tube-like concentrated vortex that dominates the most intermittent structures and contributes greatly to the singular scaling exponents. This singular scalings reflect nonsmooth or discontinuities of velocity in the limit of vanishing viscosity.

Turbulent flows consist of many different types of eddies. It is a belief that the statistics of small-scale structures in fully developed turbulence might be modeled in terms of a random distribution of different vortices. Instead of using a single type of vortex, we propose that isotropic turbulence be modeled by ensembles of various types of vortices. In this paper, we suggest that the turbulent velocity fields might be described by a combination of random distribution of Burgers vortices and Rankine vortices. Assuming a random distribution of Burgers and Rankine vortices, we calculate the statistics of turbulence by using an ensemble average of these vortices. Let $Q\left(\mathbf{v}_{B}\right)$ and $Q\left(\mathbf{v}_{R}\right)$ be the contributions to the velocity from the Burgers and the Rankine vortices, respectively. Then, the total velocity is a probability sum of $Q\left(\mathbf{v}_{B}\right)$ and $Q\left(\mathbf{v}_{R}\right): \mathbf{u}=Q\left(\mathbf{v}_{R}\right)+Q\left(\mathbf{v}_{B}\right)$. In the simplest case, the total velocity can be considered as a linear combination of these two vortices in the sense of statistics

$$
\mathbf{u}=\mathbf{v}_{B}+\lambda \mathbf{v}_{R},
$$

where $\lambda$ is a probability weighting factor. $\lambda$ depends on the ratio of the background field's dissipation to the induced velocity field's dissipation. $\lambda$ also depends on the ratio between the Rankine vortex core radius and the Burgers vortex radius. Therefore, the total velocity in the combined vortex model of Burgers and Rankine vortices (BR) is

$$
u_{\theta}=\mathrm{v}_{R, \theta}+\lambda \mathrm{v}_{B, \theta}, \quad u_{r}=-a r, \quad u_{z}=2 a z .
$$

The statistics of isotropic turbulence can be calculated using an ensemble average of the velocities in Eq. (7). The reason for using this BR model is that neither the Burgers vortex model nor the Rankine vortex model exhibits acceptable scalings for TSFs in the inertial ranges. Moreover, those two models separately do not satisfy the minimal condition for isotropic turbulence. 


\section{FORMULATIONS OF VELOCITY STRUCTURE FUNCTIONS}

In this section, we derive the LSFs and the TSFs for the $\mathrm{BR}$ vortex model. Fixing a reference point $p=(r, \theta, z)$ in the cylindrical coordinate system, the Cartesian coordinates of $p$ are

$$
x=r \cos \theta, \quad y=r \sin \theta, z .
$$

The relative position of another point $p^{\prime}$ to $p$ can be expressed using spherical coordinates $(l, \zeta, \phi)$ centered at $p$, where $\zeta$ is the polar angle and $\phi$ is the azimuthal angle. Thus, the Cartesian coordinates of $p^{\prime}$ are

$$
\begin{aligned}
& x^{\prime}=x+l \cos \phi \sin \zeta, \\
& y^{\prime}=y+l \sin \phi \sin \zeta, \\
& z^{\prime}=z+l \cos \zeta .
\end{aligned}
$$

The unit vector $\boldsymbol{l}=\left(l_{1}, l_{2}, l_{3}\right) \quad$ of $\mathbf{p p}^{\prime}$ is $(\cos \phi \sin \zeta, \sin \phi \sin \zeta, \cos \zeta)$.

Denoting the velocity differences between the two points $p$ and $p^{\prime}$ as $\delta \mathbf{u}_{l} \equiv \mathbf{u}(p)-\mathbf{u}\left(p^{\prime}\right)$, we can calculate the longitudinal and transverse velocity increments: $\delta \mathbf{u}_{l}^{L}=\left(\delta \mathbf{u}_{l} \cdot \boldsymbol{l}\right) \boldsymbol{l}$ and $\delta \mathbf{u}_{l}^{T}=\delta \mathbf{u}_{l}-\left(\delta \mathbf{u}_{l} \cdot \boldsymbol{l}\right) \boldsymbol{l}$. Thus, their magnitudes are $\delta u_{l}^{L}$ $=\left|\delta \mathbf{u}_{l}^{L}\right|$ and $\delta u_{l}^{T}=\sqrt{\delta \mathbf{u}_{l}^{T} \cdot \delta \mathbf{u}_{l}^{T}}$. For the purposes of numerical evaluation of the magnitudes, we nondimensionalize the magnitudes using $(\epsilon \nu)^{1 / 4}$

$$
\begin{aligned}
\delta u_{\hat{l}}^{L}= & \left(\frac{R_{\Gamma}^{2}}{32 \pi^{2}}+3\right)^{-1 / 4}\left[\sqrt{2} \hat{l}\left(3 \cos ^{2} \zeta-1\right)\right. \\
& \left.+\frac{R_{\Gamma}}{2 \sqrt{2} \pi} \hat{r}\left(\frac{\hat{\boldsymbol{v}}_{\theta}\left(\hat{r}^{\prime}\right)}{\hat{r}^{\prime}}-\frac{\hat{\vee}_{\theta}(\hat{r})}{\hat{r}}\right) \sin \zeta \sin \phi\right],
\end{aligned}
$$

where $\epsilon \equiv\left\langle\nu / 2\left(\partial u_{i} / \partial x_{j}+\partial u_{j} / \partial x_{i}\right)^{2}\right\rangle$ is the dissipation rate, $\hat{l}=l / r_{b}$ is the dimensionless separation, $\hat{r}_{R}=r_{R} / r_{b}$ is the dimensionless core radius, and

$$
\begin{aligned}
& \hat{l}^{\prime}=\sqrt{\hat{r}^{2}+\hat{l}^{2} \sin ^{2} \zeta+2 \hat{r} \hat{l} \sin \zeta \cos \phi,} \\
& \hat{\vee}_{\theta}(\hat{r})=\frac{1-\exp \left(-\hat{r}^{2}\right)}{\hat{r}}+\lambda \hat{\vee}_{R, \theta}(\hat{r}), \\
& \hat{\vee}_{R, \theta}(\hat{r})=\alpha \hat{r}, \quad \hat{r} \leqslant \hat{r}_{R} ; \quad \alpha \frac{\hat{r}_{R}^{2}}{\hat{r}}, \quad \hat{r}>\hat{r}_{R}, \\
& \alpha=\left(\frac{R_{\Gamma}^{2}}{32 \pi^{2}}+3\right)^{-1 / 4} \frac{R_{\Gamma}}{2 \sqrt{2} \pi} .
\end{aligned}
$$

Similarly, the magnitudes of the transverse velocity increments can be written as follows:

$$
\begin{aligned}
& \delta u_{\hat{l}}^{T}=\left(\frac{R_{\Gamma}^{2}}{32 \pi^{2}}+3\right)^{-1 / 4} \sqrt{\delta w_{1}^{2}+\delta w_{2}^{2}+\delta w_{3}^{2}}, \\
& \delta w_{i}=\sum_{j=1}^{3} w_{j}\left(\delta_{i j}-l_{i} l_{j}\right),
\end{aligned}
$$

$$
\begin{aligned}
w_{1}= & -\sqrt{2} \hat{l} \cos \phi \sin \zeta-\frac{R_{\Gamma}}{2 \sqrt{2} \pi}\left[\frac{\hat{\boldsymbol{v}}_{\theta}\left(\hat{r}^{\prime}\right)}{\hat{r}^{\prime}}(\hat{r} \sin \theta\right. \\
& \left.+\hat{l} \sin \phi \sin \zeta)-\hat{\vee}_{\theta}(\hat{r}) \sin \theta\right], \\
\omega_{2}= & -\sqrt{2} \hat{l} \sin \phi \sin \zeta+\frac{R_{\Gamma}}{2 \sqrt{2} \pi}\left[\frac{\hat{\vee}_{\theta}\left(\hat{r}^{\prime}\right)}{\hat{r}^{\prime}}(\hat{r} \cos \theta\right. \\
& \left.+\hat{l} \cos \phi \sin \zeta)-\hat{\vee}_{\theta}(\hat{r}) \cos \theta\right], \\
w_{3}= & 2 \sqrt{2} \hat{l} \cos \zeta, \\
\hat{r}^{\prime}= & \sqrt{\hat{r}^{2}+\hat{l}^{2} \sin \zeta+2 \hat{r} \hat{l} \sin \zeta \cos (\phi-\zeta) .}
\end{aligned}
$$

We note that the transverse velocity increment is not a component but an absolute value in this paper.

The ensemble averages $\langle\cdot\rangle$ in this paper are defined as spatial averages and probabilistic averages. The spatial averages are integrations over all possible orientations and locations. The probabilistic averages are the integrals, over given probability density functions of vortex Reynolds numbers and the vortex radii, see Eqs. (15) and (16).

In the DNS by Jimenéz et al. ${ }^{17}$ and the experiments by Belin et al., ${ }^{22}$ the PDF of vortex Reynolds numbers, $P\left(R_{\Gamma}\right)$, and the PDF of vortex radii, $P\left(\widetilde{r}_{b}\right)$, have been found to have exponential forms. We assume that the vortex Reynolds numbers have the exponential distribution (4). Fitting the data from Belin's experiments at higher Reynolds numbers, ${ }^{22}$ we obtain:

$$
P\left(\widetilde{r}_{b}\right)=E \widetilde{r}_{b}^{2} \exp \left(-\widetilde{r}_{b}^{0.7}\right) .
$$

Here $E^{-1}=\int_{0}^{\infty} \widetilde{r}_{b}^{2} \exp \left(-\widetilde{r}_{b}^{0.7}\right) d \widetilde{r}_{b}$ is a normalization constant and $\widetilde{r}_{b}=r_{b} / \eta$ where $\eta$ is the Kolmogorov scale. Hereafter, we will use the tilde to denote those quantities nondimensionalized by the Kolmogorov length $\eta$.

From Eqs. (10), (13), (4), and (14), the $n$th order LSFs $s_{n}^{L}(\widetilde{l})$ and TSFs $s_{n}^{T}(\widetilde{l})$ can be written as follows:

$$
\begin{aligned}
s_{n}^{L}(\widetilde{l})= & \int_{0}^{\infty} P\left(\widetilde{r}_{b}\right) d \widetilde{r}_{b} \int_{0}^{\infty} P\left(R_{\Gamma}\right) d R_{\Gamma} \int_{0}^{\widetilde{R}_{0}} d \widetilde{r} \int_{-\pi}^{\pi} d \phi \\
& \times \int_{0}^{\pi} d \zeta \frac{\widetilde{r} \sin \zeta}{2 \pi \widetilde{R}_{0}^{2}}\left(\delta u_{\tilde{l} / \widetilde{r}_{b}}^{L}\right)^{n}, \\
s_{n}^{T}(\widetilde{l})= & \int_{0}^{\infty} P\left(\widetilde{r}_{b}\right) d \widetilde{r}_{b} \int_{0}^{\infty} P\left(R_{\Gamma}\right) d R_{\Gamma} \int_{0}^{\widetilde{R}_{0}} d \widetilde{r} \int_{-\pi}^{\pi} d \phi \int_{0}^{\pi} d \zeta \\
& \times \int_{-\pi}^{\pi} d \theta \frac{\widetilde{r} \sin \zeta}{4 \pi^{2} \widetilde{R}_{0}^{2}}\left(\delta u_{\tilde{l} l \widetilde{r}_{b}}^{T}\right)^{n} .
\end{aligned}
$$

\section{NUMERICAL INTEGRATION OF THE LSF AND THE TSF}

In numerical evaluation of the BR model, there are three undetermined parameters: The weight factor $\lambda$, ratio of radii of two vortices $\hat{r}_{R}$ and the integration upper limit $\widetilde{R}_{0}$. The 


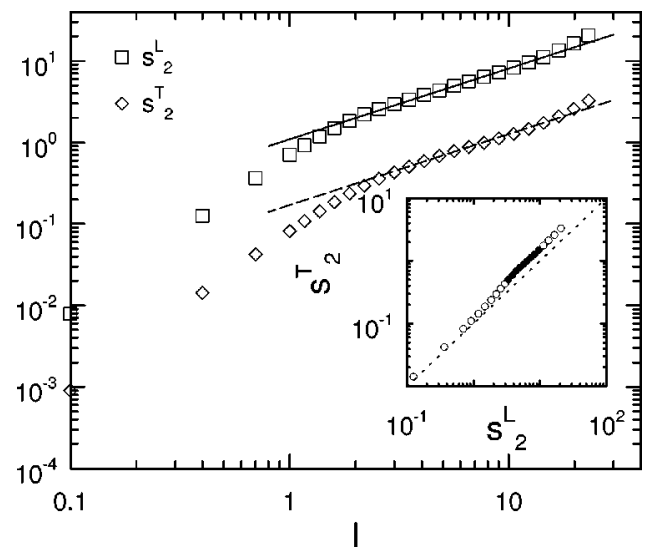

FIG. 1. The second-order transverse $(\diamond)$ and longitudinal $(\square)$ velocity structure functions as functions of the separation, $l$. The solid line is for $\sim l^{0.72}$ and the dashed line is for $\sim l^{0.71}$. The inset shows the transverse velocity structure function vs the longitudinal velocity structure function. The bullets indicate the inertial range.

weight factor and ratio of radii of two vortices can be reasonably fixed uniquely by using the constraints of isotropic turbulence. They are chosen so that the basic constraints for isotropic turbulence are satisfied: The scaling exponents of the second-order LSF and TSF are equal and the scaling exponent of the third-order LSF is equal to one. The integration upper limit $\widetilde{R}_{0}$ is determined by the constraint that ensemble averages of dissipation and enstrophy must be equal. Therefore, $\lambda=0.15, \hat{r}_{R}=0.95, \widetilde{R}_{0}=12.5$. The radius of the Rankine vortex is assumed to be related to that of the Burgers vortex. This assumption leads to a more concentrated vortex distribution near the vortex core. Since the radii of two types of vortices are related, the rate of straining of the background field has an effect on the Rankine vortex as well. The increase of Taylor-Reynolds number changes the length of the inertial range but does not change the scalings. In practical calculations, $R_{\lambda}$ is chosen to be 1290 .

A second-order trapezoidal numerical integration was used to evaluate Eqs. (15) and (16). We used 30 mesh points for each integration. Doubling the number of mesh points did not change the results reported here.

In Fig. 1, we plot the second-order LSF and TSF as functions of the separations in $\log -\log$ coordinates. Both are found to have nearly the same scaling exponents: $\tau_{2}^{L}=0.72$ and $\tau_{2}^{T}=0.71$. Therefore, the proposed BR vortex model satisfies the minimum requirement for isotropic turbulence. We also calculate the second-order LSF and TSF of the Burgers and Ranking vortex model, respectively. For the Burgers vortex model, the scaling exponent of the second-order LSF is larger than that of the second-order TSF, whereas, for the Rankine vortex model, the scaling exponent of LSF is less than that of the TSF. The Rankine vortex has a smaller contribution to the LSFs but a larger contribution to the TSFs. This can be seen from Eqs. (15) and (16).

In Fig. 2, we present the third-order LSF and TSF. Their scaling exponents are approximately equal to unity. Therefore, the BR model satisfies the basic constraint of isotropic turbulence.

In Fig. 3, the $n$ th-order LSFs and TSFs for $n=4,6,8$,

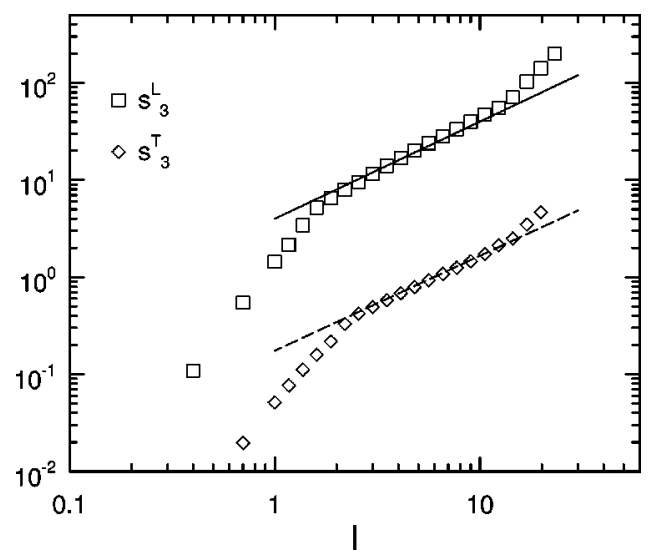

FIG. 2. The third-order transverse $(\diamond)$ and longitudinal $(\square)$ velocity structure functions as functions of the separation, $l$. The solid line and dashed line are for $\sim l$.

and 10 are shown. It is observed that there exist two scaling ranges. The first scaling range corresponds to the dissipation range and the second scaling range corresponds to the inertial range. To compare the scaling exponents $\tau_{n}^{L}$ and $\tau_{n}^{T}$ of the LSFs and the TSFs in the inertial range, we plot $s_{n}^{T}$ versus $s_{n}^{L}$ for $n=6,8$ in Fig. 4. It is easy to see that their slopes are less than unity, which indicates different scaling exponents for finite inertial ranges.

The scaling exponents $\tau_{n}^{L}$ and $\tau_{n}^{T}$ can be evaluated using linear regression of the LSFs and TSFs. In Fig. 5, $\tau_{n}^{L}$ and $\tau_{n}^{T}$ are plotted and compared with the results from DNS. Qualitatively, they show that the $\tau_{n}^{L}$ are larger than the $\tau_{n}^{T}$ for $n$ $>2$.

\section{DISCUSSIONS AND CONCLUSIONS}

In this paper, we have discussed the velocity structure functions using a combined vortex model (BR). The vorticity field in the BR model is composed of Burgers and Rankine vortices. The second-order velocity structure functions of the BR model can be shown to satisfy the minimal condition for isotropic turbulence. Their LSFs and TSFs exhibit satisfactory scalings and demonstrate a transition from the dissipa-

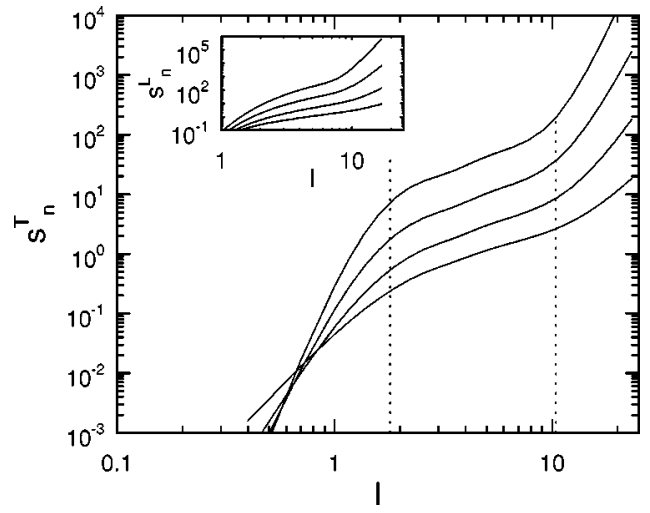

FIG. 3. The transverse velocity structure functions as functions of the separation $l$, for $n=4,6,8,10$ from bottom to top. The inertial range is within the two dotted lines. The inset shows similar relations for the longitudinal structure functions. 


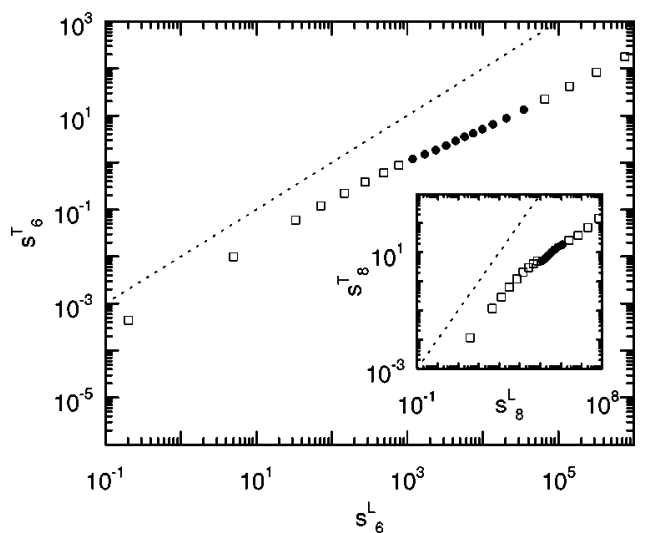

FIG. 4. The relative scaling of the sixth-order transverse structure function vs the sixth-order longitudinal structure function. The inset shows the relative scaling for the eighth-order quantities. The bullets indicate the inertial range quantities and the slopes of dotted lines are unity.

tion range to the inertial range. However, neither the Burgers nor the Rankine vortex model satisfies the isotropic condition. Actually, neither the TSFs from the Burgers vortex model nor the LSFs from the Rankine vortex model exhibit an acceptable inertial range. The anomalous scaling is the result of vortex intermittency. Our model contains strongly intermittent filaments and nonuniform spatial distributions of vortices. Both of them make contributions to the anomalous scaling.

The present results show significant differences between the high-order $(n>4)$ scaling exponents for the LSFs and TSFs. However, the scaling of the fourth-order LSF seems not much different from the corresponding TSF which is in agreement with experimental measurements by Arad et al. ${ }^{23}$ This suggests that the high-order LSFs and the TSFs have different intermittent intensities, associated with intermittent structures. The most intermittent structures in isotropic turbulence have been observed to be filaments in DNS and experiments. ${ }^{24-28}$ The filaments can be described mathematically using Burgers and/or Rankine vortices, ${ }^{16}$ which have different intermittent intensities. The BR model contains strong intermittent filaments and a nonuniform spatial distribution of vortex intensities. Both of them make contributions to the anomalous scaling. From Eqs. (15) and (16), the con-

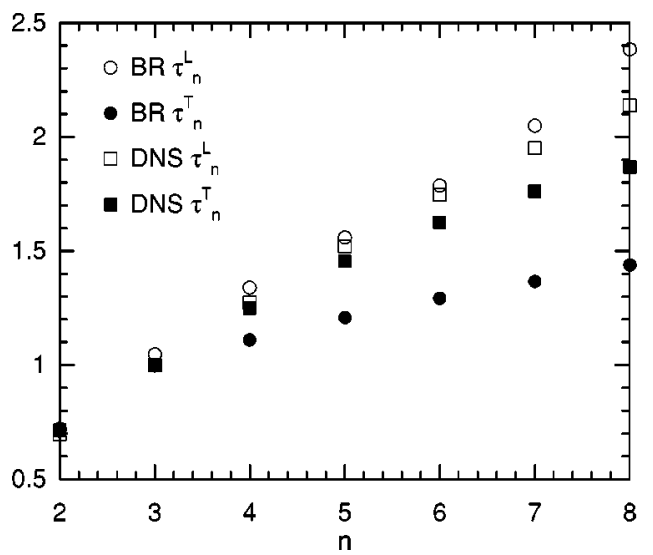

FIG. 5. Scaling exponents of the velocity structure functions. tributions of the Burgers and Rankine vortices to the LSFs differ significantly from their contributions to the TSFs. It is this difference that produces the different scaling exponents of the LSFs and the TSFs. This conclusion is supported by recent results: ${ }^{7,9}$ The intermittent parameters in the hierarchy models are different for velocity structure functions of longitudinal and transversal directions.

In principal, the present model is not only valid for finite Reynolds number, but also for infinite Reynolds number. However, only finite Reynolds numbers are accessible to numerical integrations. Calculations can only be done for large but finite Reynolds number. Therefore, our conclusions are valid for large enough Reynolds number but not for infinite Reynolds number. In actual turbulence and turbulent simulations, the observed vortex structures have a variety of forms. The current model is an over-simplified model of turbulence. However, since the BR model captures the underlying physics of the intermittent structures, such as vortex filaments, it provides some understanding of different scaling exponents of isotropic fields.

${ }^{1}$ A. N. Kolmogorov, "The local structure of turbulence in incompressible viscous fluid for very large Reynolds numbers," C. R. Acad. Sci. URSS 30, 301 (1941).

${ }^{2}$ K. R. Sreenivasan and R. A. Antonia, "The phenomenology of small-scale turbulence,"’ Annu. Rev. Fluid Mech. 29, 435 (1997).

${ }^{3}$ J. A. Herweijer and W. Van de Water, "Transverse structure functions of turbulence," in Advances in Turbulence $V$, edited by R. Benzi (Kluwer, Dordrecht, 1995), pp. 210-216.

${ }^{4}$ R. Camussi, D. Barbagallo, R. Guj, and F. Stella, "Transverse and longitudinal scaling laws in non-homogeneous low Re turbulence,' Phys. Fluids 8, 1181 (1996).

${ }^{5}$ H. Kahalerras, Y. Malecot, and Y. Gagne, "Transverse velocity structure functions in developed turbulence," in Advances in Turbulence VI, edited by S. Gavrilakis, L. Michiels, and P. A. Monkewitze (Kluwer, Dordrecht, 1996), pp. 235-238.

${ }^{6}$ R. Camussi and R. Benzi, "Hierarchy of transverse structure functions," Phys. Fluids 9, 257 (1997).

${ }^{7}$ O. N. Boratav and R. B. Pelz, "Structures and structure functions in the inertial range of turbulence," Phys. Fluids 9, 1400 (1997); O. N. Boratav,

"On longitudinal and lateral moment hierarchy in turbulence," ibid. 9, 3120 (1997).

${ }^{8}$ A. Noullez, G. Wallace, W. Lempert, R. B. Miles, and U. Frisch, "Transverse velocity increments in turbulent flow using the RELIEF technique," J. Fluid Mech. 339, 287 (1997).

${ }^{9}$ S. Grossmann, D. Lohse, and A. Reeh, "Different intermittency for longitudinal and transversal turbulent fluctuations," Phys. Fluids 9, 3817 (1997).

${ }^{10}$ S. Chen, K. R. Sreenivasan, M. Nelkin, and N. Z. Cao, "A refined similarity hypothesis for transverse structure functions,' Phys. Rev. Lett. 79, 2253 (1997); "Inertial range scalings of dissipation and enstrophy in isotropic turbulence," 79, 1253 (1997).

${ }^{11}$ R. A. Antonia and B. R. Pearson, "Scaling exponents for turbulent velocity and temperature increments," Europhys. Lett. 40, 123 (1997).

${ }^{12}$ B. Dhruva, Y. Tsuji, and K. R. Sreenivasan, "Transverse structure functions in high-Reynolds-number turbulence," Phys. Rev. E 56, R4928 (1997).

${ }^{13}$ G. W. He, S. Y. Chen, R. H. Kraichnan, R. Y. Zhang, and Y. Zhou, "Statistics of dissipation and enstrophy induced by localized vortices," Phys. Rev. Lett. 81, 4636 (1998).

${ }^{14}$ J. L. Synge and C. C. Lin, "On a statistical model of isotropic turbulence," Trans. R. Soc. Can. 37, 45 (1943).

${ }^{15} \mathrm{~J}$. M. Burgers, "A mathematical model illustrating the theory of turbulence,'” Adv. Appl. Mech. 1, 45 (1948).

${ }^{16}$ H. K. Moffatt, S. Kida, and K. Ohkitani, "Stretched vortices-the sinews of turbulence; large-Reynolds-number asymtotics,' J. Fluid Mech. 266, 371 (1994).

${ }^{17}$ J. Jiménez, A. A. Wray, P. G. Saffman, and R. S. Rogallo, “The structure 
of intense vorticity in isotropic turbulence," J. Fluid Mech. 255, 65 (1993).

${ }^{18}$ P. G. Saffman and D. I. Pullin, "Calculation of velocity structure functions for vortex models of isotropic turbulence,', Phys. Fluids 8, 3072 (1996).

${ }^{19}$ N. Hatakeyama and T. Kambe, "Statistical laws of random strained vortices in turbulence,' Phys. Rev. Lett. 79, 1257 (1997).

${ }^{20}$ H. Lamb, Hydrodynamics (Dover, New York, 1932).

${ }^{21}$ T. Kambe and I. Hosokawa, "Statistics of small-scale structures and a dynamical mechanism of cascade,' in Small-Scale Structures in ThreeDimensional Hydrodynamic and Magnetohydrodynamic Turbulence, edited by M. Meneguzzi, A. Pouquet, and P-L. Sulem (Springer, New York, 1995), pp. 123-130.

${ }^{22}$ F. Belin, J. Maurer, P. Tabeling, and H. Willaime, "Observation of intense filaments in fully-developed turbulence,'’ J. Phys. II 6, 573 (1996).
${ }^{23}$ I. Arad, B. Dhruva, S. Kurien, V. S. L'vov, I. Procaccia, and K. R. Sreenivasan, "Extraction of anisotropic contributions in turbulent flows," Phys. Rev. Lett. 81, 5330 (1998).

${ }^{24}$ E. D. Siggia, "Numerical study of small-scale intermittency in threedimensional turbulence,' J. Fluid Mech. 107, 375 (1981).

${ }^{25}$ R. M. Kerr, "Higher-order derivative correlations and the alignment of small-scale structures in isotropic numerical turbulence,', J. Fluid Mech. 153, 31 (1985).

${ }^{26}$ Z. S. She, E. Jackson, and S. A. Orszag, "Intermittent vortex structures in homogeneous isotropic turbulence,' Nature (London) 344, 226 (1990).

${ }^{27}$ A. Vincent and M. Meneguzzi, "The spatial structure and statistical properties of homogeneous turbulence,'” J. Fluid Mech. 225, 1 (1991).

${ }^{28}$ S. Douady, Y. Couder, and M. E. Brachet, "Direct observation of the intermittency of intense vorticity filaments in turbulence,' Phys. Rev. Lett. 67, 983 (1991). 